\title{
EXTENSIONS OF BRANDT SEMIGROUPS
}

\author{
BY R. J. WARNE
}

\author{
Communicated by O. G. Harrold, February 21, 1966
}

The purpose of this note is to announce the determination of all (ideal) extensions of a Brandt semigroup by an arbitrary semigroup with zero and to give two applications of this result. We use the terminology and notation of [1].

THEOREM. Let $(V$, o) be an extension of a Brandt semigroup $S$ by an arbitrary semigroup $T$ with zero, $0^{\prime}$. Let $S$ be given the Rees representation $S=M^{0}(G ; I, I ; \Delta)$. Then there exists a partial homomorphism $w: A \rightarrow w_{A}$ of $T^{*}=\left(T \backslash 0^{\prime}\right)$ into $g_{I}$ the full symmetric inverse semigroup on $I$. Let $s_{A}$ and $t_{A}$ denote the domain and range of $w_{A}$ respectively. If $A B=0^{\prime}$ (juxtaposition denoting multiplication in $T$ ) either $s_{A} \cap t_{B}=\square$ or $s_{A} \cap t_{B}$ is a single element $d_{A, B}$. For each $A \in T^{*}$ there exists a mapping $\psi_{A}$ of $s_{A}$ into the group $G$ such that for $A B=0^{\prime}$

$$
\left(i \psi_{A}\right)\left(i w_{A} \psi_{B}\right)=i \psi_{A B} \quad \text { for all } i \in s_{A B} .
$$

The products in $V$ are given by

$$
\begin{aligned}
& \text { (a) } A \circ B=A B \text { if } A B=0^{\prime} \text { in } T, \\
& \text { (b) } A \circ B=0 \text { (in } S \text { ) if } A B=0^{\prime} \text { (in } T \text { ) and } t_{A} \cap s_{B}=\square, \\
& \text { (c) } \left.A \circ B=\left(d_{A, B} w_{A}^{-1} \psi_{A}\right)\left(d_{A, B} \psi_{B}\right) ; d_{A, B} w_{A}^{-1}, d_{A, B} w_{B}\right) \\
& \text { if } A B=0^{\prime} \text { (in } T \text { ) and } t_{A} \cap s_{B}=d_{A, B} .
\end{aligned}
$$

(2) $(a ; i, j) \circ A= \begin{cases}\left(a\left(j \psi_{A}\right) ; i, j w_{A}\right) & \text { if } j \in s_{A}, \\ 0 & \text { if } j \bar{\in} s_{A},\end{cases}$

$$
0 \circ A=0
$$

(3) $A \circ(a, i, j)= \begin{cases}\left(\left(i w_{A}^{-1} \psi_{A}\right) a ; i w_{A}^{-1}, j\right) & \text { if } i \in t_{A}, \\ 0 & \text { if } i \bar{\in} t_{A},\end{cases}$

$$
A \circ 0=0 .
$$

Conversely let $S$ be a Brandt semigroup and $T$ be a semigroup with zero such that $S \cap T=\square$. If we are given the mappings w and $\psi_{A} d e-$ scribed above and define product 0 in the class sum of $S$ and $T^{*}$ by (1)-(3), then $V$ is an extension of $S$ by $T$.

Remark. An extension of $S$ by $T$ always exists [1]. 
CoRollary 1. An extension $V$ of a Brandt semigroup $S$ by a regular 0-bisimple semigroup $T$ is given by a partial homomorphism [2, p. 522] if and only if there exists an idempotent $E$ in $T^{*}$ such that there is at most one idempotent of $S^{*}$ under $E$.

REMARK. Warne [2] gave a similar result for the case where $S$ and $T$ are both completely 0 -simple semigroups.

If $A$ is a set, $|A|$ will denote the cardinality of $A$.

CoRollary 2. If $S$ is a finite Brandt semigroup and $T^{*}$ is a simple group with $\left|T^{*}\right|>\max (|I| !|G|)$, then there are $2^{|I|}$ extensions of $S$ by $T$.

These results will appear in [3].

\section{REFERENCES}

1. A. H. Clifford and G. B. Preston, The algebraic theory of semigroups, Vol. 1, Math. Surveys No. 7, Amer. Math. Soc., Providence, R. I., 1961.

2. R. J. Warne, Extensions of completely 0-simple semigroups by completely 0simple semigroups, Proc. Amer. Math. Soc. 17 (1966), 522-523.

3. - Extensions of Brandt semigroups and applications (to appear).

West Virginia University 